\title{
TACE or TARE for Unresectable Neuroendocrine Liver Metastases: Can we Finally Start to Focus on Value?
}

\author{
Charles R. Scoggins, MD, MBA
}

The Hiram C. Polk Jr, MD Department of Surgery, University of Louisville School of Medicine, Louisville, KY

Physicians who treat patients with liver tumors frequently encounter neuroendocrine liver metastases (NELM). Resection is the best choice because it confers the best survival advantage. Many patients, however, will not be resectable, so alternative therapies are used. Hepatic arterial therapy, which involves percutaneous delivery of therapeutic particles to the tumor or tumors in a minimally invasive fashion, is a common nonsurgical local therapy. "Hepatic arterial therapy" (HAT) is a catch-all term that denotes a variety of treatments including bland embolization, chemoembolization, drug-eluting bead chemoembolization, and radioembolization. But which treatment should be used? At the moment, it appears that chemoembolization and radioembolization both are relatively safe, well-tolerated, and effective in helping to control both the disease burden and hormone-related symptoms.

With little meaningful comparative data available, the choice of which method to use is clouded with personal bias and local preference. Some hospitals offer only bland embolization or transarterial chemoembolization (TACE), whereas others prefer transarterial radioembolization (TARE).

Further complicating the issue, TACE often is a catchall term that can mean a variety of methods. At some centers, TACE involves the use of lipiodol, chemotherapy, and embolic particles that interrupt blood flow. At other centers, TACE refers to drug-eluting bead TACE (DEB-

(C) Society of Surgical Oncology 2021

First Received: 2 December 2020

Accepted: 5 January 2021;

Published Online: 28 January 2021

C. R. Scoggins, MD, MBA

e-mail: Charles.scoggins@louisville.edu
TACE). The small beads are loaded with chemotherapy agents that elute the drug within the microvasculature of the tumor.

The data comparing conventional TACE with DEBTACE for NELM are sparse. One retrospective study compared conventional TACE, DEB-TACE, and TARE for patients with NELM and found a survival benefit for conventional TACE compared with both DEB-TACE and TARE. ${ }^{1}$ The data from this study are somewhat limited in their impact because they are retrospective and single-institutional. Although intriguing, these data are by no means definitive. Furthermore, it appears that DEB-TACE might be associated with a higher complication rate than conventional TACE.

Other studies have sought to find survival differences between TACE and TARE. One large, multi-institutional study found comparably low complication rates and the same overall survival rates between TACE and TARE. ${ }^{2}$ The data in this study are in contrast to the results (in favor of TACE) reported by Minh et al. ${ }^{1}$ We simply do not know for sure which method is more effective. Many experts and experienced clinicians who see these patients on a regular basis reserve TARE for patients with symptoms of hormone overproduction because the radioembolization appears to be particularly effective in reducing (and in some patients, abrogating) hormone production.

We all strive to alleviate symptoms and improve survival for our patients. Ideally, these aims are accomplished while minimizing cost (this is the concept of value). To really understand value, we must have a grip on the financial data. Both TACE and TARE involve multiple phases of care, and multiple costs are incurred, some fixed and some variable.

In a small, nonrandomized series of NELM by Whitney et al. ${ }^{3}$ the issue of cost was examined. In their study, 15 patients who received 23 TARE treatments were compared 
with 28 patients who underwent 26 TACE treatments using drug-eluting beads loaded with doxorubicin. After 1 year, the two groups differed little in terms of response rates, but the median cost in the TARE group $(\$ 25,243)$ was nearly twice that in the TACE group $(\$ 13,400)$. As is commonplace, the TACE patients spent at least one night in the hospital, whereas the TARE patients were treated as outpatients. ${ }^{3}$ Surely, this has an impact on cost, as does the variable costs of mapping procedures, shunt calculations, professional fees of additional providers (e.g., radiation oncologists), and the delivery vehicles themselves.

Similarly, Egger et al. ${ }^{2}$ reported that the TACE patients had a longer hospital stay than the TARE patients. Nearly all the TACE patients spent a night in the hospital, and because the majority of the TACE procedures in this study were performed at one of the participating hospitals, this practice might reflect an institutional bias. ${ }^{2}$ Although cost data were not reported, it can be extrapolated that postprocedure admission will have an impact on the finances involved. We and others have increasingly moved to perform TACE selectively as an outpatient procedure, with good tolerance. The common post-TACE side effects of fever, abdominal pain, and nausea can be managed reliably with oral medications. These practices need to be standardized.

In this issue of Annals of Surgical Oncology, Ngo et al. ${ }^{4}$ report the largest to-date effort comparing chemoembolization (TACE) with radioembolization (TARE) for patients with unresectable neuroendocrine liver metastases. ${ }^{4}$ This well-done article describes a meta-analysis that studied data from six retrospective studies on NELM. In all six studies, the data seem to favor TACE for survival benefit. The authors noted similar tumor burden and tumor grade among all the studies. The three studies that included RECIST data found no difference in the treatment responses. No financial data are presented, so value cannot be assessed. The data in this article are the most compelling to date and should help us frame the next set of questions for study.

Like all good studies, this meta-analysis ${ }^{4}$ serves to guide clinicians and pique the interest of investigators. Questions arise, and it is apparent that we need a series of real randomized controlled trials focusing on survival (perhaps progression-free survival is a reasonable end point for a disease that has a very long natural history), patient-centered end points such as quality of life, and value. Stratification needs careful consideration to ensure as much parity as possible, and efforts should concentrate on standardization of charge data to provide some sense of the finances involved. Given the available data, it seems that TACE is a reasonable choice for patients with unresectable NELM who require therapy.

\section{REFERENCES}

1. Minh DD, Chapiro J, Gorodetski B, Huang Q, Liu C, Smolka S, et al. Intra-arterial therapy of neuroendocrine tumor liver metastases: comparing conventional TACE, drug-eluting beads TACE, and yttrium-90 radioembolization as treatment options using a propensity score analysis model. Eur Radiol. 2017;27:4995-5005.

2. Egger ME, Armstrong E, Martin RCG, Scoggins CR, Philips P, Shah M, et al. Transarterial chemoembolization vs radioembolization for neuroendocrine liver metastases: a multi-institutional analysis. JACS. 2020;200:363-70.

3. Whitney R, Valek V, Fages JF, et al. Transarterial chemoembolization and selective internal radiation for the treatment of patients with metastatic neuroendocrine tumors: a comparison of efficacy and cost. Oncologist. 2011;16:594-601.

4. Ngo L, Elnahla A, Attia AS, Hussein M, Toraih EA, Kandil E, Killackey M. Chemoembolization versus radioembolization for neuroendocrine liver metastases: a meta-analysis comparing clinical outcomes. Ann Surg Oncol. 2021. https://doi.org/10.1245 /s10434-020-09469-4.

Publisher's Note Springer Nature remains neutral with regard to jurisdictional claims in published maps and institutional affiliations. 